\title{
Design, Synthesis and Photophysical Properties of New 2,6-Dicyanoanilines Based on Isophthalaldehyde and Terephthalaldehyde Skeleton
}

\author{
ANANADA S. KUDALE ${ }^{* 1,4}$, NAGANATH G. PATIL ${ }^{2}$, SANTOSH B. KAMBLE ${ }^{3}$, \\ SHOBHA V. RUPANAR ${ }^{1}$ and VASANT B. HELAVI ${ }^{1}$ \\ ${ }^{1}$ Department of Chemistry, Rajaram College, Kolhapur 416004, Maharashtra, India \\ ${ }^{2}$ CSIR-National Chemical Laboratory, Dr. Homi Bhabha Road, Pashan, \\ Pune 411008, Maharashtra, India \\ ${ }^{3}$ Department of Chemistry, Yashwantrao Chavan Institute of Science, \\ Satara 415002, Maharashtra, India \\ ${ }^{4}$ Directorate of Forensic Science Laboratories, Kalina, Santacruz (E), \\ Mumbai 400098, Maharashtra, India \\ as.kudale@gmail.com
}

Received 9 February 2016 / Accepted 26 February 2016

\begin{abstract}
A novel series of 2, 6-dicyanoanilines based on isophthalaldehyde and terephthalaldehyde were designed, synthesized and characterized by spectral methods. The new chemical entities thus synthesized were studied for their photophysical properties.
\end{abstract}

Keywords: Isophthalaldehyde, Terephthalaldehyde, Dicyanoanilines, Malanonitrile

\section{Introduction}

The 2,6-dicyanoanilines and their analogues are known for their significant fluorescent properties ${ }^{1}$ and have been studied and exploited in many different areas of science like nonlinear optical materials ${ }^{2}$, molecular electronic devices ${ }^{3}$ and have been reported to exhibit biological activities like antileishmanial ${ }^{4}$ and antifungal ${ }^{5}$ activity. Also, the flexibility of conversion of cyano and amino groups in to other functional groups makes these compounds versatile for utilization as intermediates in the preparation of many diverse substrates for comprehensive use. There are number of such compounds and methods of their preparations are reported in the literature ${ }^{6,7}$. Most of the reported 2,6-dicyanoanilines and their related compounds are based on single Acceptor-Donor-Acceptor (A-D-A) moiety on the aromatic skeleton except for a few synthetic strategies reported for tri-substituted 2,6-dicyanoanilines by Klebe ${ }^{8}$, Webster et al., ${ }^{9}$ and Wallenfels et al., ${ }^{10}$ where single aromatic ring bears two A-D-A systems. 
To the best of our knowledge, there is no report on the multicomponent reaction of aromatic di-aldehyde (phthalaldehyde, isophthalaldehyde or terephthalaldehyde) with malononitrile and aliphatic aldehyde in the presence of base which can deliver a number of very interesting new chemical entities and the structure and property, relationship of this type of molecules can be studied. H. B. Borate and group ${ }^{11}$ reported the multicomponent reaction of aromatic aldehyde, aliphatic aldehyde and malononitrile in the presence of morpholine in dimethylformamide to give 3-aryl,4-alkyl-2,6-dicyanoanillnes in good yield.

\section{Experimental}

We designed molecules based on the same synthetic strategy to build up new chemical entities bearing two A-D-A systems (Scheme 1 and 2, Table 1). Accordingly, we performed multicomponent reactions of isophthalaldehyde or terephthalaldehyde with malononitrile and aliphatic aldehyde (butanal or hexanal or nonanal) in the presence of morpholine in dimethylformamide at $80{ }^{\circ} \mathrm{C}$ for $8 \mathrm{~h}$ and results are presented herein. As expected, the reaction gave mixture of a number of products and it was difficult to isolate the desired compound by column chromatography. The desired products were obtained by recrystallization of crude product followed by column chromatography. All the products were characterized by ${ }^{1} \mathrm{H}$ NMR, ${ }^{13} \mathrm{C}$ NMR, IR and mass spectroscopy. These compounds were found to be soluble in DMF and DMSO and sparingly soluble in methanol, acetonitrile, chloroform, acetone, dichloromethane, ethyl acetate etc.

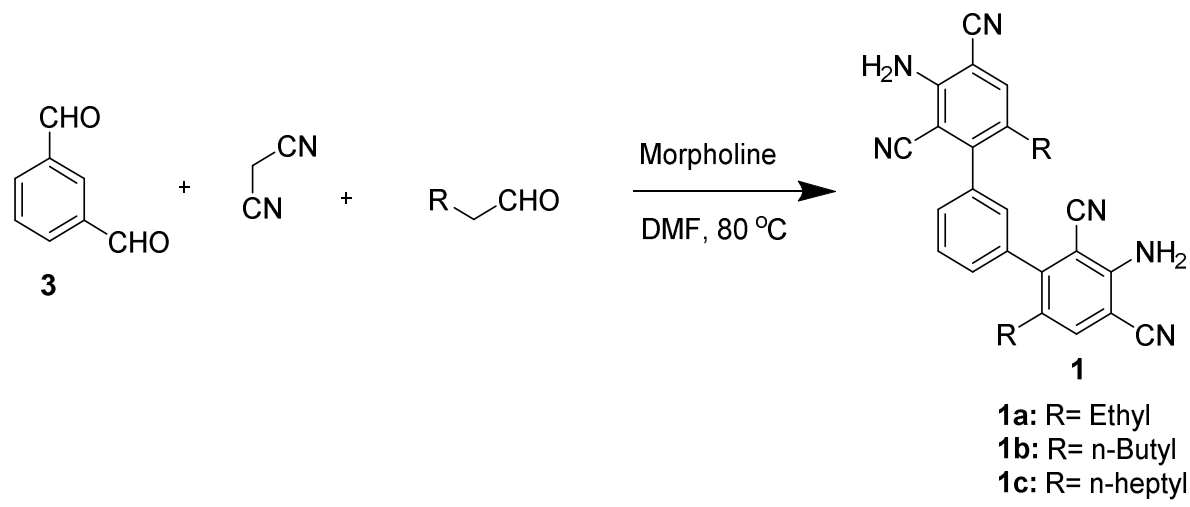

Scheme 1. Synthesis of dicyanoanilines based on isophthalaldehyde skeleton<smiles>O=Cc1ccc(C=O)cc1</smiles><smiles>[R]C[CH]O</smiles><smiles>N#CCC#N</smiles>

4<smiles>[R]c1cc(C#N)c(N)c(C#N)c1-c1ccc(-c2c([R])cc(C#N)c(N)c2C#N)cc1</smiles>

2

Scheme 2. Synthesis of dicyanoanilines based on terephthalaldehyde skeleton 
Table 1. Synthesis of new 2,6-dicyanoanilines (1a-1c and 2a-2c)

\begin{tabular}{ccccc}
\hline Entry & $\begin{array}{c}\text { Aliphatic } \\
\text { aldehyde }\end{array}$ & Aromatic aldehyde & Product & Yeild $^{\mathrm{a}}$ \\
\hline 1 & Butanal & 3 & $1 \mathrm{a}$ & 35 \\
2 & Hexanal & 3 & $1 \mathrm{~b}$ & 43 \\
3 & Nonanal & 3 & $1 \mathrm{c}$ & 50 \\
4 & Butanal & 4 & $2 \mathrm{a}$ & 45 \\
5 & Hexanal & 4 & $2 \mathrm{~b}$ & 48 \\
6 & Nonanal & 4 & $2 \mathrm{c}$ & 53 \\
\hline
\end{tabular}

${ }^{a}$ The yields given are for isolated products

Representative procedure for the preparation of 3,3''-diamino-6,6''-diheptyl[1,1':3',1',-terphenyl]-2,2', 4,4',-tetracarbonitrile (1c)

To a mixture of isophthalaldehyde $(0.500 \mathrm{~g}, 0.003727 \mathrm{~mol})$, nonanal $(1.272 \mathrm{~g}, 0.008946$ $\mathrm{mol})$ and malononitrile $(1.08 \mathrm{~g}, 0.01639)$ in dry DMF taken in a round bottom flask and equipped with reflux condenser and guard tube, morpholine $(1.55 \mathrm{~g}, 0.01788 \mathrm{~mol})$ was added at $0{ }^{\circ} \mathrm{C}$ and the mixture was allowed to cool to room temperature and stirred at 80 ${ }^{\circ} \mathrm{C}$ for $8 \mathrm{~h}$. Progress of the reaction was checked by the TLC. (Solvent system- Petroleum ether: Ethyl acetate $(80: 20))$. After completion of reaction, the reaction mixture was allowed to cool to room temperature and water was added, then extracted with excess of ethyl acetate $(250 \mathrm{~mL})$, dried over sodium sulphate and concentrated on rotavapour. Ethyl acetate $(10 \mathrm{~mL})$ was added and the solution of reaction mixture was added slowly drop by drop to $100 \mathrm{~mL}$ petroleum ether for recrystallization. After settlement of the precipitate in the beaker, the supernatant solution was decanted in other beaker. The precipitate thus obtained was washed with $10 \%$ ethyl acetate and petroleum ether mixture. The residue thus obtained was chromatographed on silica gel (60-120 mesh) using $15-20 \%$ ethyl acetate in petroleum ether to afford 3,3' '-diamino-6,6' -diheptyl[1,1':3',1' '-terphenyl]-2,2', 4,4' '-tetracarbonitrile as off-white flappy solid (1.03 g 50\% ) mp: $190{ }^{\circ} \mathrm{C}$. UV (In DMF): 361nm. IR (Neat): 1270, 1470, 1556, 1591, 1643, 2218, 2853, 2923, 2953, 3243, 3336, 3442, $3476 \mathrm{~cm}^{-1}$. ${ }^{1} \mathrm{H}$ NMR (200 MHz, CDCl $): 0.46-0.73$ $(\mathrm{m}, 6 \mathrm{H}), 0.77-1.28(\mathrm{~m}, 20 \mathrm{H}), 1.99-2.28(\mathrm{~m}, 4 \mathrm{H}), 4.95$ and $5.08(2 \mathrm{~s}, 4 \mathrm{H}), 6.90(\mathrm{~s}, 1 \mathrm{H})$, $7.14(\mathrm{~d}, 2 \mathrm{H}), 7.30(\mathrm{~s}, 2 \mathrm{H}), 7.42(\mathrm{t}, 1 \mathrm{H}) .{ }^{13} \mathrm{C}$ NMR $\left(50 \mathrm{MHz}, \mathrm{CDCl}_{3}\right): 14.01(2 \mathrm{C})$, $22.51(2 \mathrm{C}), \quad 28.86(4 \mathrm{C}), \quad 30.79(2 \mathrm{C}), \quad 31.59(2 \mathrm{C}), \quad 31.90(2 \mathrm{C}), \quad 96.66(2 \mathrm{C}), \quad 98.80(2 \mathrm{C})$, 115.18(2C), 115.95(2C), 128.43, 128.91(2C), 129.17(2C), 131.52, 137.25 (2C), 137.42(2C), 149.26(2C), 149.53(2C). MS (ESI) $m / z: 579.2(\mathrm{M}+\mathrm{Na})$. HRMS Obtained $\mathrm{C}_{36} \mathrm{H}_{41} \mathrm{~N}_{6} 557.3400(\mathrm{M}+\mathrm{H})$, Calculated $\mathrm{C}_{36} \mathrm{H}_{41} \mathrm{~N}_{6} 557.3393(\mathrm{M}+\mathrm{H})$. (In case of $1 \mathbf{a}$ and $2 \mathrm{a}$ the amount of base (Morpholine) used was 3.0 equivalent)

3,3' '-Diamino-6,6' '-dibutyl-[1,1':3',1' '-terphenyl]-2,2',,4,4' '-tetracarbonitrile (1b)

Off-white flappy solid (0.709 g 43\%) mp: $212{ }^{\circ} \mathrm{C}$. UV (In DMF): $361 \mathrm{~nm}$. IR (Neat): $1269,1477,1664,2164,2218,2870,2929,2956,3243,3336,3443,3480 \mathrm{~cm}^{-1} .{ }^{1} \mathrm{H}$ NMR $\left(500 \mathrm{MHz}, \mathrm{CDCl}_{3}+\mathrm{DMSO}_{6} \mathrm{~d}_{6}\right): 0.62-0.77(\mathrm{~m}, 6 \mathrm{H}), 0.98-1.15(\mathrm{~m}, 4 \mathrm{H}), 2.20-2.37(\mathrm{~m}, 4 \mathrm{H})$, $6.60(\mathrm{~s}, 4 \mathrm{H}), 7.20(\mathrm{~s}, 1 \mathrm{H}), 7.38-7.47(\mathrm{~m}, 2 \mathrm{H}), 7.62-7.70(\mathrm{~m}, 1 \mathrm{H}), 7.75(\mathrm{~s}, 1 \mathrm{H}), 7.76(\mathrm{~s}$, 1H). ${ }^{13} \mathrm{C}$ NMR (125 MHz, DMSO-d $\left.{ }_{6}\right): 13.58(2 \mathrm{C}), 21.66(2 \mathrm{C}), 30.90$ (2C), 32.41 (2C), 95.65 (2C), 97.71 (2C), 115.47 (2C), 116.47 (2C), 128.37 (1C), 128.64 (2C), 128.94 (1C), 129.31 (2C), 137.45 (2C), 138.41 (2C), 149.33 (1C), 149.61 (1C), 150.61 (1C), 150.76 (1C). HRMS Obtained $\mathrm{C}_{30} \mathrm{H}_{29} \mathrm{~N}_{6} 473.2457(\mathrm{M}+\mathrm{H}), \mathrm{C}_{30} \mathrm{H}_{29} \mathrm{~N}_{6}$ Calculated 473.2355(M+H)). 


\section{3,3"-Diamino-6,6"-diethyl-[1,1':3',1"-terphenyl]-2,2",4,4"-tetracarbonitrile (1a)}

Off-white flappy solid $(0.542 \mathrm{~g}, 35 \%) \mathrm{mp}: 22{ }^{\circ} \mathrm{C}$. UV (DMSO): $361 \mathrm{~nm}$. IR (Neat): 1273, 1469, 1550, 1594, 1647, 2220, 2857, 2924, 2953, 3242, 3334, 3442, $3445 \mathrm{~cm}^{-1}$. ${ }^{1} \mathrm{H}$ NMR (400 MHz, CDCl $\left.\mathrm{CDMSO}_{3}+\mathrm{d}_{6}\right)$ : 0.04-0.16 (m, 6H), 1.39-1.56 (m, 4H), $5.25(\mathrm{~s}$, $4 \mathrm{H}), 6.27(\mathrm{~s}, 1 \mathrm{H}), 6.45-6.54(\mathrm{~m}, 2 \mathrm{H}), 6.68(\mathrm{~s}, 2 \mathrm{H}), 6.73-6.81(\mathrm{~m}, 1 \mathrm{H}) .{ }^{13} \mathrm{C}$ NMR $(100$ MHz, $\mathrm{CDCl}_{3}+\mathrm{DMSO}_{-} \mathrm{d}_{6}$ ): 13.80 (2C), 23.55 (2C), 94.89 (2C), 96.77 (2C), 114.03 (2C), 114.94 (2C), 127, 127.12 (2C), 127.51 (2C), 129.28, 135.73 (2C), 136.17 (2C), 147.48, 147.73, 149.18, 149.29. HRMS Obtained $\mathrm{C}_{26} \mathrm{H}_{21} \mathrm{~N}_{6} 417.1822(\mathrm{M}+\mathrm{H})$, Calculated $\mathrm{C}_{26} \mathrm{H}_{21} \mathrm{~N}_{6} 417.1829(\mathrm{M}+\mathrm{H})$.

\section{3,3"-Diamino-6,6"-diheptyl-[1,1':4',1"-terphenyl]-2,2",4,4"-tetracarbonitrile (2c)}

Off-white flappy solid $(1.09 \mathrm{~g}, 53 \%) \mathrm{mp}$ : decomposition around $240-243{ }^{\circ} \mathrm{C}$. UV (DMF): $361 \mathrm{~nm}$. IR (Neat): 1267, 1466, 1644, 2219, 2851, 2921, 2952, 3226, 3324, 3378, 3444, $3469 \mathrm{~cm}^{-1} .{ }^{1} \mathrm{H}$ NMR $\left(200 \mathrm{MHz}, \mathrm{CDCl}_{3}\right): 0.84(\mathrm{t}, 6 \mathrm{H}), 1.03-1.47(\mathrm{~m}, 20 \mathrm{H}), 2.37(\mathrm{t}, 4 \mathrm{H}), 5.15$ (bs, 4H), 7.39(s, 4H), 7.53(s, 2H). ${ }^{13} \mathrm{C}$ NMR $\left(125 \mathrm{MHz}, \mathrm{CDCl}_{3}+\mathrm{DMSO}_{6}\right): 13.72(2 \mathrm{C})$, 21.99 (2C), 28.23, 28.37 (2C), 28.47, 28.62, 30.34, 30.44, 31.09, 31.14, 31.48, 95.85 (2C), 97.68, 97.93, $115.08(2 \mathrm{C}), 116.04(2 \mathrm{C}), 128.31(2 \mathrm{C}), 128.35$ (2C), 129.30, 129.48, 137.09, $137.13,137.35,137.43,148.89,149.13,150.33,150.46$. HRMS Obtained $\mathrm{C}_{36} \mathrm{H}_{41} \mathrm{~N}_{6}$ $557.3397(\mathrm{M}+\mathrm{H}) \mathrm{C}_{36} \mathrm{H}_{41} \mathrm{~N}_{6}$ Calculated $557.3394(\mathrm{M}+\mathrm{H})$.

\section{3,3"-Diamino-6,6"-dibutyl-[1,1':4',1"-terphenyl]-2,2",4,4"-tetracarbonitrile (2b)}

Off-white flappy solid $(0.792 \mathrm{~g}, 48 \%) \mathrm{mp}$ : decomposition around $267-270{ }^{\circ} \mathrm{C}$. UV (DMF): $361 \mathrm{~nm}$. IR (Neat): 1266, 1466, 1632, 2216, 2865, 2928, 2953, 3237, 3358, $3477 \mathrm{~cm}^{-1} .1 \mathrm{H}$ NMR (500 MHz, DMSO-d 6 ): 0.70 (bs, 6H), 1.06 (bs, 4H), 1.24 (bs, 4H), 2.27 (bs, 4H), 6.62 (bs, 4H), 7.44 (bs, 4H), 7.78 (bs, 2H). ${ }^{13} \mathrm{C}$ NMR (125 MHz, DMSO-d 6 ): $13.48(2 \mathrm{C}), 21.69$ (2C), 30.95 (2C), 32.48 (2C), 95.64 (2C), 97.47, 97.71, 115.43 (2C), 116.48 (2C), 128.62 (2C), 128.71 (2C), 129.09, 129.20, 137.19, 137.32, 138.53 (2C), 149.57, 149.83, 150.81, 150.70. MS (ESI) $m / z: 495.2(\mathrm{M}+\mathrm{Na})$. HRMS Obtained $\mathrm{C}_{30} \mathrm{H}_{29} \mathrm{~N}_{6}: 473.2448(\mathrm{M}+\mathrm{H})$, Calculated $\mathrm{C}_{30} \mathrm{H}_{29} \mathrm{~N}_{6}: 473.2455(\mathrm{M}+\mathrm{H})$.

\section{3,3"-Diamino-6,6"-diethyl-[1,1':4',1"-terphenyl]-2,2",4,4"-tetracarbonitrile (2a)}

Off-white flappy solid $(0.697 \mathrm{~g}, 45 \%) \mathrm{mp}$ : decomposition around $277-278{ }^{\circ} \mathrm{C}$. UV (DMF): $361 \mathrm{~nm}$. IR (Neat): 1267, 1463, 1632, 2215, 2863, 2930, 2951, 3238, 3358, $3478 \mathrm{~cm}^{-1} .{ }^{1} \mathrm{H}$ NMR (500 MHz, DMSO-d $\left.{ }_{6}\right)$ : 0.81-1.00 (m, 6H), 2.20-2.37 (m, 4H), $6.63(\mathrm{~s}, 4 \mathrm{H}), 7.45$ and $7.46(2 \mathrm{~s}, 4 \mathrm{H}), 7.79$ and $7.80(2 \mathrm{~s}, 2 \mathrm{H}) .{ }^{13} \mathrm{C}$ NMR $(125 \mathrm{MHz}$, DMSO-d 6 ): 14.87, 15.02, 24.59, 24.67, 95.77 (2C), 97.44, 97.58, 115.50 (2C), 116.49 (2C), 128.63 (2C), 128.68 (2C), 128.76, 129.54, 137.14, 137.30, 138.00 (2C), 149.39, 149.59, 150.73, 150.84 . HRMS Obtained $\mathrm{C}_{26} \mathrm{H}_{21} \mathrm{~N}_{6} 417.1822(\mathrm{M}+\mathrm{H})$, Calculated $\mathrm{C}_{26} \mathrm{H}_{21} \mathrm{~N}_{6} 417.1829(\mathrm{M}+\mathrm{H})$ ).

\section{Results and Discussion}

\section{Photophysical properties}

The photophysical properties of synthesized compounds were studied as they show strong fluorescence under UV. The UV-Visible absorption and fluorescence spectra of synthesized compounds were recorded in dimethyl formamide and are presented in Figure 1 and 2. The wavelength of maximum absorption $\left(\lambda_{\max }\right)$ in the UV was observed at $\sim 360 \mathrm{~nm}$ while the wavelength of maximum emission $\left(\lambda_{\text {max.emi }}\right)$ for the compounds was observed at $\sim 412 \mathrm{~nm}$. The fluorescence spectra of the target compounds were recorded at fixed UV absorption of 0.1 optical densities. 
Table 2. UV and fluorescence observations of 1a-1c and 2a-2c

\begin{tabular}{cccc}
\hline $\begin{array}{c}\text { Compd. } \\
\text { No. }\end{array}$ & $\begin{array}{c}\text { UV absorption } \\
\text { maximum at } 361 \mathrm{~nm}\end{array}$ & $\begin{array}{c}\text { Fluorescence } \\
\text { maximum at } 412 \mathrm{~nm}\end{array}$ & $\begin{array}{c}\text { Length of carbon } \\
\text { chain }\end{array}$ \\
\hline 1a & 0.1021 & 34.32 & Two \\
1b & 0.1000 & 36.83 & Four \\
1c & 0.1030 & 33.39 & Seven \\
2a & 0.1078 & 20.21 & Two \\
2b & 0.1068 & 27.61 & Four \\
2c & 0.1035 & 25.37 & Seven \\
\hline
\end{tabular}

The compound 1b showed maximum fluorescence and the compound 2a showed minimum fluorescence while all other entities showed intermediate fluorescence. The values for fluorescence maxima of compounds with various chains are given in Table 2 and it is observed that between the two isomers of phthalaldehyde used in present study, the compound obtained from the isophthalaldehyde with four carbon chain shows maximum fluorescence.

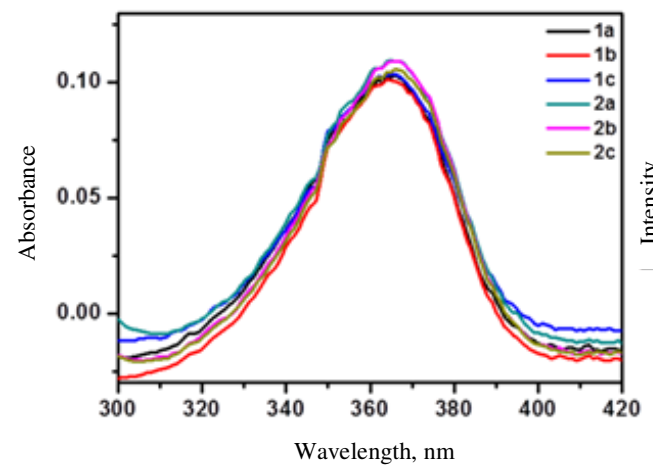

Figure 1. UV Abosorption Spectra of compound 1a-c, 2a-c

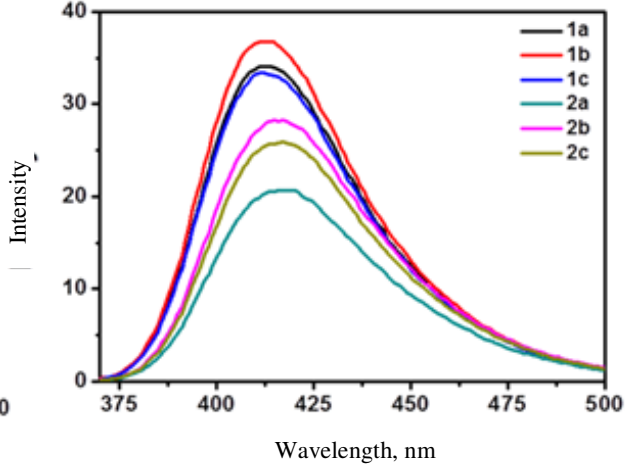

Figure 2. Fluorescence spectra of compounds 1a-c, 2a-c

\section{Conclusion}

This work describes the preliminary results about synthesis of novel 2,6-dicyanoanilines obtained from aromatic dialdehydes, isophthalaldehyde and terephthalaldehyde and their fluorescence properties. These compounds can be modified to obtain a variety of useful compounds and can be screened for various applications. Based on these results, we are planning to synthesize compounds with long hydrophobic aliphatic chains and polar groups in the molecule and to screen these molecules in different forensic applications like finding adulteration in the vegetable oil and staining the fingerprints etc.

\section{Acknowledgement}

We thank Government of Maharashtra, India and Principal, Y. C. Institute of Science, Satara, Maharashtra, India for their support.

\section{References}

1 Cui S L, Lin X F and Gang W Y, J Org Chem., 2005, 70(7), 2866-2869; DOI:10.1021/jo047823h

2 (a) Nalwa H S, Adv Mater., 1993, 5(5), 341-358; DOI:10.1002/adma.19930050504

(b) Kanis D R, Ratner M A and Marks T J, Chem Rev., 1994, 94(1), 195-242;

DOI:10.1021/cr00025a007 (c) Long N J, Angew Chem., Int Ed Engl., 1995, 34(1), 21-38; 
DOI:10.1002/anie.199500211 (d) Wong M S, Bosshard C, Pan F and Gunter P, Adv Mater., 1996, 8(8), 677-680; DOI:10.1002/adma.19960080818 (e) Cardozo T M and Nascimento M A C, J Mater Sci., 2005, 40(13), 3549-3551; DOI:10.1007/s10853005-2883-x

3 Van Mullekom H A M, Vekemans J A J and Meijer E W, Chem Eur J., 1998, 4(7), 1235-1243;DOI:10.1002/(SICI)1521-3765(19980710)4:7<1235::AID-

CHEM1235>3.0.CO;2-4 (b) Carroll R L and Gorman C B, Angew Chem., Int Ed Engl., 2002, 41(23), 4378-4400; DOI:10.1002/1521-3773(20021202)41:23<4378::AIDANIE4378>3.0.CO;2-A (b) Bendikov M, Wudl F and Perepichka D F, Chem Rev., 2004, 104(11), 4891-4946; DOI:10.1021/cr030666m

4 Singh F V, Vatsyayan R, Roy U and Goel A, Bioorg Med Chem Lett., 2006, 16(10), 2734-2737; DOI:10.1016/j.bmcl.2006.02.012

5 Borate H B, Kudale A S, Chavan S P, Kunte S S, Chandavarkar M A, Iyer R, Tawte A C and Rao D D, WO 2014132267 A1 (2014).

6 Borate H B, Kudale A S and Agalave S G, Org Prep Proced Int., 2012, 44, 467-521; DOI:10.1080/00304948.2012.715055

7 Ramulu J B, Chanda T, Chowdhury S, Nandib G C and Singh M S, RSC Adv., 2013, 3, 5345-5349; DOI:10.1039/C3RA40450A (b) Mohammadi B, Shafieey M, Kazemi H and Ramazani A, Chinese Chemi Lett., 2013, 24(6), 497-499; DOI:10.1016/j.cclet.2013.03.046 (c) Datta B and Pasha M A, J Chem Sci., 2013, 125(2), 291-294; DOI:10.1007/s12039-013-0375-0 (d) Mohammadi B, Kazemi H and Shafieey M, Monatshefte fur Chemie-Chemical Monthly, 2014, 145, 1649-1652; DOI: 10.1007/s00706-014-1229-2; (e) Molla A and Hussain S, RSC Adv., 2014, 4, 2975029758; DOI:10.1039/C4RA03627A (f) Wei C and Xiao-Yan L, Chemical Papers, 2015, 69(7), 1016-1020; DOI:10.1515/chempap-2015-0098; (g) Yalcin E, Kutlu Y C, Korkmaz V and Sahin E, ARKIVOK, 2015 (v), 202-2018; (h) Zhang Y L, Li Y F, Shi Y K, Yu B, Zhang G C, Qi P P, Fu D J, Shan L H and Liu H M, Steroids, 2015, 104, 1-7; DOI:10.1016/j.steroids.2015.07.005 (i) Safaei-Ghomi J, Zahedi S, Javid M and Ghasemzadeh M A, JNS, 2015, 5(2), 153-160.

8 Klebe J F, J Am Chem Soc., 1964, 86, 3399-3400; DOI:10.1021/ja01070a044

9 Webster O W, Brown M and Benson R E, J Org Chem., 1965, 30(9), 3250-; DOI:10.1021/jo01020a535

10 Wallenfels K, Witzler F and Friedrich K, Tetrahedron, 1967, 23(4), 1845-1855; DOI:10.1016/S0040-4020(01)82585-0

11 Sawargave S P, Kudale A S, Deore J V, Bhosale D S, Divse J M, Chavan S P and Borate H B, Tetrahedron Lett., 2013, 54(12), 1528-1530; DOI:10.1016/j.tetlet.2013.01.008 